\title{
Revue illustrée des principales indications de CBCT en orthodontie.
}

\author{
Auteurs: \\ Olszewski R DDS, MD, $\mathrm{PhD}, \mathrm{DrSc}^{1,2^{*}}$ \\ Theys S DDS ${ }^{3}$, \\ Perez E DDS ${ }^{4}$, \\ Wisniewska K $\mathrm{DDS}^{5}$, \\ Wisniewski M DDS, MD, $\mathrm{PhD}^{6}$
}

\author{
Affiliations: \\ ${ }^{1}$ Department of Oral and maxillofacial surgery, Cliniques universitaires saint Luc, \\ UCLouvain, Brussels, Belgium \\ ${ }^{2}$ Oral and maxillofacial surgery research Lab, NMSK, IREC, SSS, UCLouvain, \\ Brussels, Belgium \\ ${ }^{3}$ Department of pediatric dentistry, Cliniques universitaires saint Luc, UCLouvain, \\ Brussels, Belgium \\ ${ }^{4}$ Department of orthodontics, Cliniques universitaires saint Luc, UCLouvain, \\ Brussels, Belgium \\ ${ }^{5}$ Orthodontic private practice, Orthos, Meander street 22, Warsaw, Poland \\ ${ }^{6}$ Clinical department of maxillofacial surgery, Military Institute of Medicine, \\ Szaserów street 128, Warsaw, Poland \\ *Corresponding author: Pr R. Olszewski, Department of oral and maxillofacial \\ surgery, Cliniques universitaires saint Luc, Université catholique de Louvain,
}


Brussels, Belgium, phone+3227645718; fax: +3227645876; ORCID iD:orcid.org/0000-0002-2211-7731

Disclaimer: the views expressed in the submitted article are our own and not an official position of the institution or funder. 


\section{Summary}

Cette revue illustrée porte sur les principales indications actuellement recommandées dans la littérature d'utilisation du cone beam computed tomography (CBCT) en orthodontie. Il s'agit des anomalies dentaires, des canines incluses, des dents surnuméraires, des troubles de l'éruption et des résorptions radiculaires externes liées aux traitements orthodontiques. L'examen CBCT doit être justifié individuellement, au cas par cas, et de pouvoir apporter un bénéfice au patient en terme de diagnostic et/ou de traitement orthodontique. L'orthodontiste prescripteur doit être capable d'interpréter et est responsable de l'interprétation de tout ce qui est visible sur l'ensemble du champs de vue du CBCT.

Mots-clés: CBCT, orthodontie, anomalie dentaire, canine incluse, dent surnuméraire, troubles d'éruption, résorption radiculaire externe

This illustrated article is reviewing currently accepted and recommended indications for use of cone beam computed tomography (CBCT) in orthodontics. We present CBCT cases of teeth anomalies, impacted canines, supernumerary teeth, troubles of teeth eruption, and external roots resorption related to orthodontic teeth movements. The CBCT should be justified for each patient, and should present added value for diagnostic and/or for treatment planning. The orthodontist needs to interpret and is responsible for all of the field of view.

Keywords: CBCT, orthodontics, dental anomalies, impacted canine, supernumerary teeth, eruption troubles, external roots resorption 


\section{Introduction}

La tomographie à faisceau conique (cone beam computed tomography, CBCT) existe en dentisterie depuis 2001 [1]. La rotation du couple générateur de faisceau conique - détecteur autour de la tête du patient permet de balayer l'ensemble du champs de vue choisi en une seule rotation sans interpolation [1]. Les images issues $\mathrm{du}$ CBCT permettent la reconstruction multiplanaire en trois dimensions et une visualisation selon les trois plans de l'espace: axial, sagittal et coronal. Elles offrent également la possibilité de visualiser une téléradiographie de face, de profil et une radiographie panoramique reconstituée. Le CBCT opère dans le système DICOM $[1]$.

Plusieurs indications cliniques d'utilisation du CBCT en orthodontie sont apparues dans la littérature scientifique. Celles-ci respectent les principes de radioprotection ALARA (As Low As Reasonably Achievable) et, plus récemment, ALADAIP (As Low As Diagnostically Acceptable being Indication-oriented and Patient-specific) $[2,3]$.

L'American Academy of Oral and Maxollofacial Radiology (AAOMR) et la British Othodontic Society recommandent l'utilisation du CBCT uniquement dans les cas spécifiques pour lesquels la radiographie en deux dimensions est insuffisante à l'établissement du diagnostic [4]. Les indications reconnues sont les fentes labioalvéolo-palatines, les dents incluses, les dents surnuméraires et la chirurgie orthognatique (planning) [4]. D'autres indications cliniques du CBCT en orthodontie ont été proposées dans le rapport du projet européen DIMITRA (dentomaxillofacial paediatric imaging: an investigation toward low-dose radiation induced risk) de 2018. Il s'agit des anomalies dentaires et des résorptions radiculaires externes [4, 5].

L'orthodontie concerne majoritairement une population pédiatrique. L'importance des principes de base de radioprotection, que sont la justification (indication correcte), l'optimisation (ex; choix adéquat du champs de vue) et la limitation de la dose, est d'autant plus grande [4].

La connaissance des aspects techniques de l'équipement CBCT utilisé est capitale en ce qui concerne l'optimisation et la réduction de la dose. En effet, selon le modèle et la marque de la machine utilisée, certains paramètres d'exposition (comme le $\mathrm{kV}$, le $\mathrm{mA}$ et le temps d'exposition) sont prédéfinis et non ajustables pour un patient donné [4]. En l'absence d'adaptation des paramètres d'irradiation au patient pédiatrique, la dose d'irradiation de l'enfant peut dépasser celle de l'adulte suite à la différence de taille de leurs organes ainsi qu'à leur susceptibilité aux rayonnements [4]. Dès lors, il est préconisé d'utiliser le champs de vue le plus petit disponible en fonction de l'indication afin de réduire la dose d'irradiation $[4,6]$. 
Cette revue illustrée portera sur les principales indications recommandées d'utilisation du CBCT en orthodontie: les anomalies dentaires, les canines incluses, les dents surnuméraires, les troubles de l'éruption et les résorptions radiculaires externes liées aux traitements orthodontiques [1,5].

\section{Principales indications}

\section{Anomalies dentaires}

Le CBCT permet d'évaluer la forme, la taille et le volume des dents, y compris des racines. Les caractéristiques particulières de ces dernières influencent le traitement orthodontique par les appareils fixes et peuvent parfois être un obstacle à la réalisation de celui-ci. La morphologie des racines est, dans certains cas, un facteur favorisant la survenue de résorption externe par l'application d'un excès de force dans la mobilisation de ces dents [1]. Deux variations morphologiques des racines sont illustrées ci-dessous: le nombre de racines et la dilacération radiculaire. La figure 1 montre la différence de visualisation du nombre de racines d'une même dent, la première molaire mandibulaire droite, entre la reconstruction tridimensionelle (3D) (A) et la vue axiale (B) issue des images du CBCT. La dilacération radiculaire d'une deuxième prémolaire mandibulaire gauche est illustrée sur la figure 2.

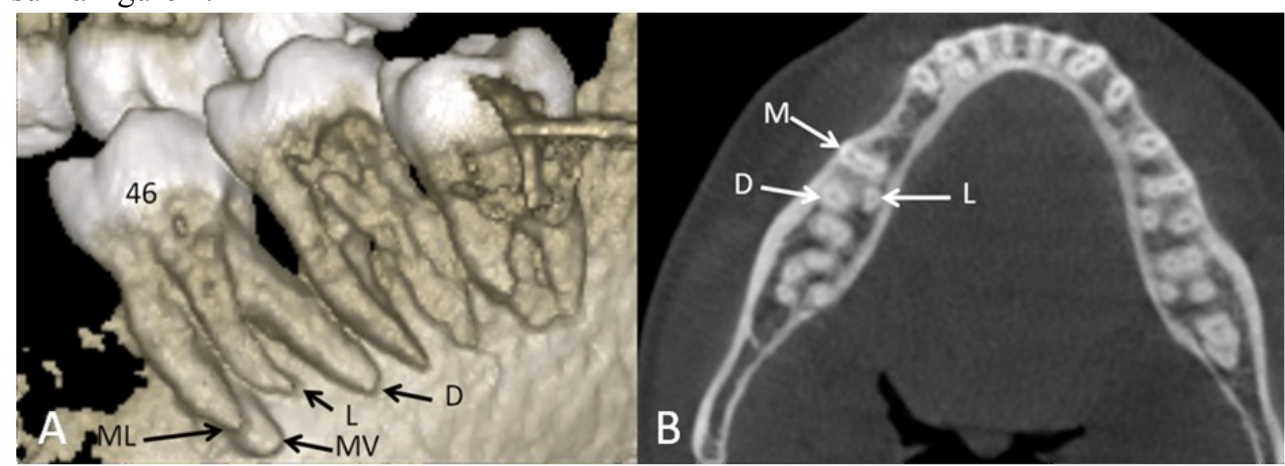

Fig. 1. A. Reconstruction 3D, vue linguale du secteur postérieur droit de l'arcade dentaire inférieure. La première molaire mandibulaire droite (dent 46) présente quatre racines: mésio-vestibulaire (MV), mésio-linguale ( $M L)$, linguale $(L)$ et distale $(D)$. $B$. Vue axiale du même cas. La première molaire mandibulaire droite (dent 46$)$ présente trois racines: mésiale $(M)$, linguale $(L)$ et distale (D). 


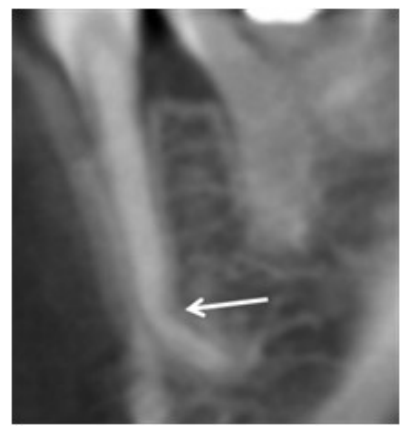

Fig. 2. Vue sagittale. La flèche indique la dilacération de la racine de la deuxième prémolaire mandibulaire gauche (dent 35 ).

\section{Canines incluses}

Le CBCT permet de visualiser et d'estimer, en trois dimensions, la position des canines incluses ainsi que de mesurer l'angulation de son grand axe par rapport aux trois plans de l'espace (axial, sagittal et coronal) [5,7]. Cependant, il n'existe actuellement, aucun système standardisé permettant de décrire précisément la position des canines par rapport aux trois plans de l'espace [8]. Selon la position de la canine incluse et l'extension du sac folliculaire de celle-ci, les dents adjacentes peuvent être atteintes de résorption radiculaire. Ce risque de résorption radiculaire peut être estimé sur les images du CBCT [9]. Une attention particulière doit être portée sur la vérification de la surface des racines de l'incisive centrale, de l'incisive latérale (la plus fréquemment atteinte par ce type de résorption) et de la première prémolaire. Cette indication d'utilisation du CBCT en orthodontie est illustrée par deux situations cliniques. La première est celle d'une jeune fille de 14 ans présentant une canine maxillaire gauche incluse palatine. La figure 3 permet de visualiser cette dent incluse ainsi que ses rapports avec les structures voisines. Le deuxième cas clinique est celui d'un garçon de 14 ans avec une canine maxillaire gauche incluse vestibulaire (figure 4). 


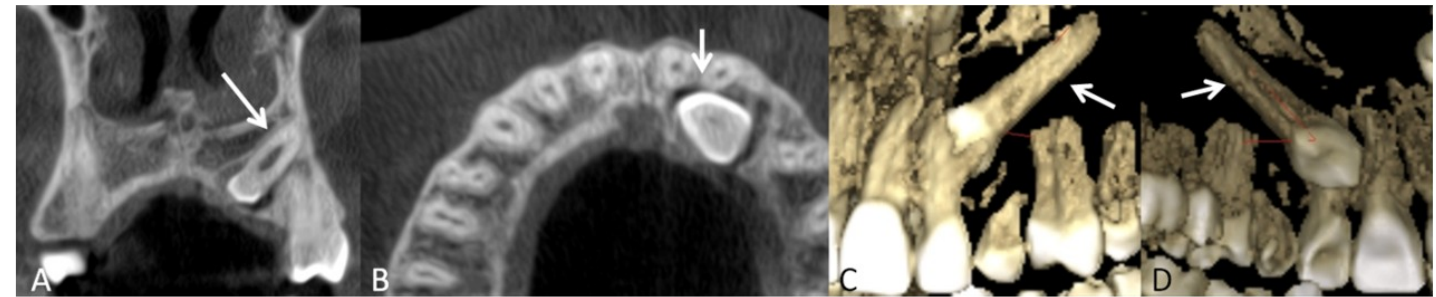

Fig. 3. A. Vue frontale. Canine maxillaire gauche incluse (dent 23, flèche), en palatin, son grand axe est dirigé vers caudal et médial.

B. Vue axiale. La couronne de la dent 23 incluse et palatine est en contact étroit avec la paroi palatine de la racine de l'incisive latérale maxillaire gauche (dent 22, flèche). Le sac folliculaire autour de la couronne de la dent 23 est en contact avec la paroi palatine de l'incisive centrale maxillaire gauche (dent 21).

C. Reconstruction 3D, vue antéro-latérale gauche. Canine maxillaire gauche incluse (dent 23, flèche), palatine, dont le grand axe est dirigé vers caudal et médial, située à distance des racines de la première prémolaire maxillaire gauche (dent 24).

D. Reconstruction 3D, vue postéro-latérale gauche. La pointe canine de la dent 23 incluse (flèche) se trouve en palatin entre les racines des incisives centrale (dent 21) et latérale (dent 22) maxillaires gauches.

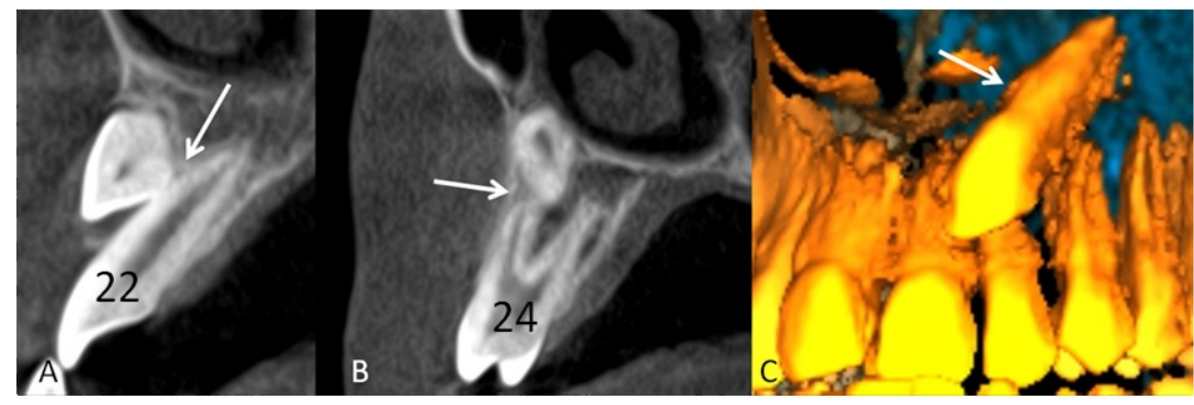

Fig. 4. A. Vue sagittale. La flèche indique le contact étroit entre la couronne de la canine maxillaire gauche incluse et la racine de l'incisive latérale maxillaire gauche (dent 22).

B. Vue sagittale. La flèche montre le contact étroit entre la racine de la 23 et la racine vestibulaire de la première prémolaire maxillaire gauche (dent 24). C. Reconstruction 3D, vue antéro-latérale gauche. Canine maxillaire gauche incluse en vestibulaire (dent 23 , flèche). 


\section{Dents surnuméraires}

Préalablement à tout traitement orthodontique, il est important de diagnostiquer la présence éventuelle de dents surnuméraires car celles-ci peuvent être à l'origine de résorptions radiculaires iatrogènes [10] ou de troubles de l'éruption des dents voisines. Le CBCT offre la possibilité de visualiser la présence de dents surnuméraires mais également leur localisation, leur morphologie (forme, taille et volume) et leur position par rapport aux dents et structures nobles à proximité. Il permet ainsi d'évaluer le risque de résorption radiculaire externe ou de lésions des structures nobles en cas d'avulsion [11]. Le CBCT permet aussi d'expliquer de potentiels troubles d'éruption liés à la présence d'une dent surnuméraire [11]. Cette situation est présentée par le cas clinique d'un garçon de 8 ans dans les figures 5 et 6. La mésiodens est la dent surnuméraire la plus fréquente. Elle est localisée sur la ligne médiane entre les incisives centrales maxillaires [12]. La figure 7 illustre la présence d'une mésiodens chez un garçon de 10 ans.

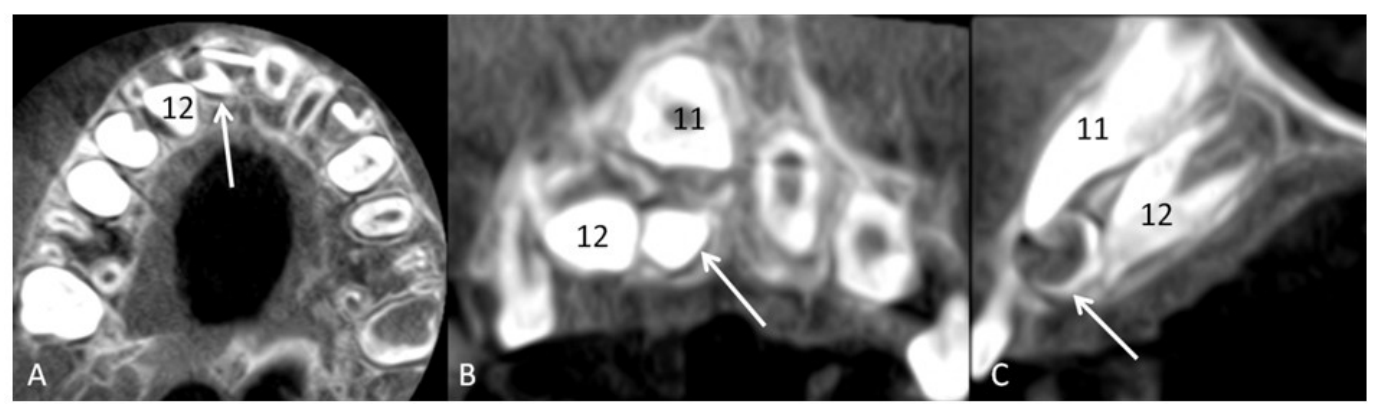

Fig. 5. A. Vue axiale. La flèche montre la présence d'une dent surnuméraire en palatin de l'incisive centrale maxillaire droite (dent 11). L'incisive latérale maxillaire droite (dent 12) est en retrait, en palatin.

B. Vue frontale. La dent surnuméraire (flèche) est localisée au rebord alvéolaire en caudal de la dent 11 et en médial par rapport à la dent 12 . C. Vue Sagittale. L'éruption de l'incisive centrale maxillaire droite est bloquée par la dent surnuméraire (flèche). Celle-ci est également responsable de la position palatine de l'incisive latérale maxillaire droite. 


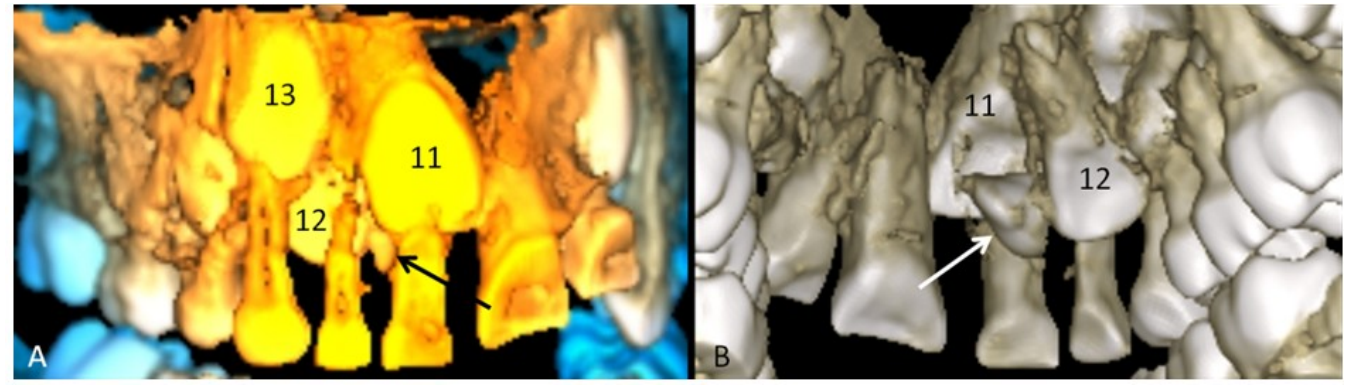

Fig. 6. A. Reconstruction 3D, vue antérieure. La dent surnuméraire (flèche) est partiellement visible sous le rebord incisif de l'incisive centrale maxillaire droite (dent 11) incluse. L'incisive latérale maxillaire droite (dent 12) incluse est située en palatin. Les dents temporaires sont présentes sur l'arcade. B. Reconstruction 3D, vue palatine. La dent surnuméraire (flèche) empêche l'éruption de la dent 11 par sa face mésiale et de la dent 12 par sa face distale.

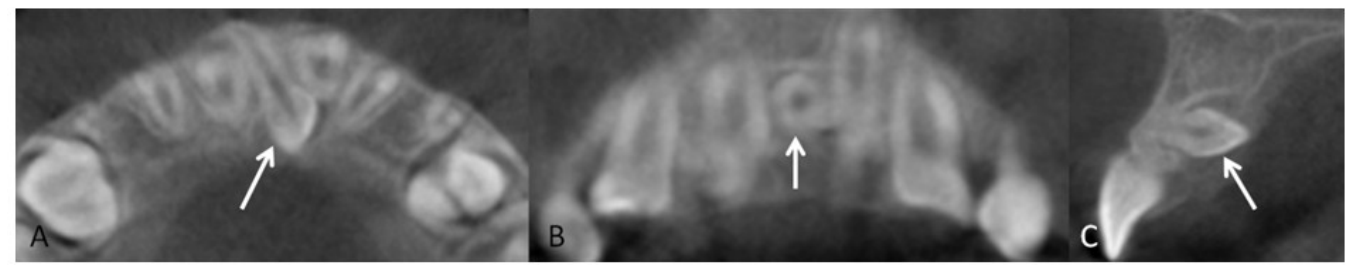

Fig. 7. A. Vue axiale. Présence d'une mésiodens (flèche) dont la couronne est dirigée vers la gauche et vers dorsal.

B. Vue frontale. La mésiodens est à l'horizontal (flèche). Sa présence engendre la formation d'un diastème entre les incisives centrales maxillaires. C. Vue sagittale: La couronne de la mésiodens, entourée de son sac folliculaire (flèche), perfore la table osseuse palatine et soulève la muqueuse palatine.

\section{Troubles d'éruption}

Les raisons des retards d'éruption ou de la non-éruption de dents sur l'arcade ne sont pas toujours simples à identifier. L'association des visualisations en deux dimensions et en trois dimensions issues du CBCT permet de mieux comprendre le processus de ces troubles d'éruption. Lorsque ces derniers sont unilatéraux, il est important de comparer les images du coté atteint à celles du coté sain afin de mieux cerner la dynamique des événements. Le retard d'éruption de l'incisive latérale mandibulaire droite chez un garçon de 9 ans est illustrée à la figure 8. Les images du CBCT d'un garçon de 15 ans ayant un retard d'éruption de la deuxième molaire 
maxillaire droite ainsi que la comparaison avec celle de la dent controlatérale sont présentées à la figure 9 .

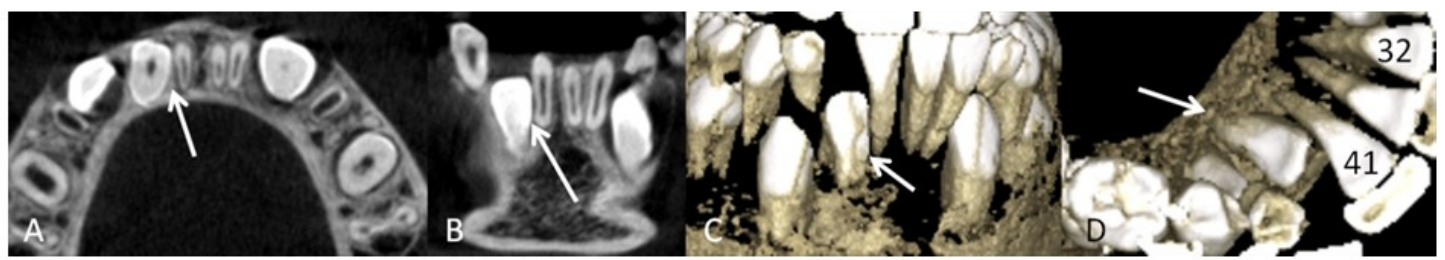

Fig. 8. A. Vue axiale. Retard d'éruption de l'incisive latérale mandibulaire droite (dent 42, flèche). Celle-ci est légèrement en lingual et en contact avec la racine de l'incisive centrale mandibulaire droite (dent 41) et avec le sac folliculaire de la canine mandibulaire droite (dent 43) incluse en vestibulaire. B. Vue frontale. La flèche montre la dent 42 incluse en position verticale. C. Reconstruction 3D, vue antéro-latérale droite. L'incisive latérale mandibulaire droite incluse et en rotation vers la ligne médiane (flèche). D. Reconstruction 3D, vue occlusale. La largeur de la racine de la 42 incluse (flèche) est différente (plus grande) que celle de l'incisive latérale controlatérale (dent 32).

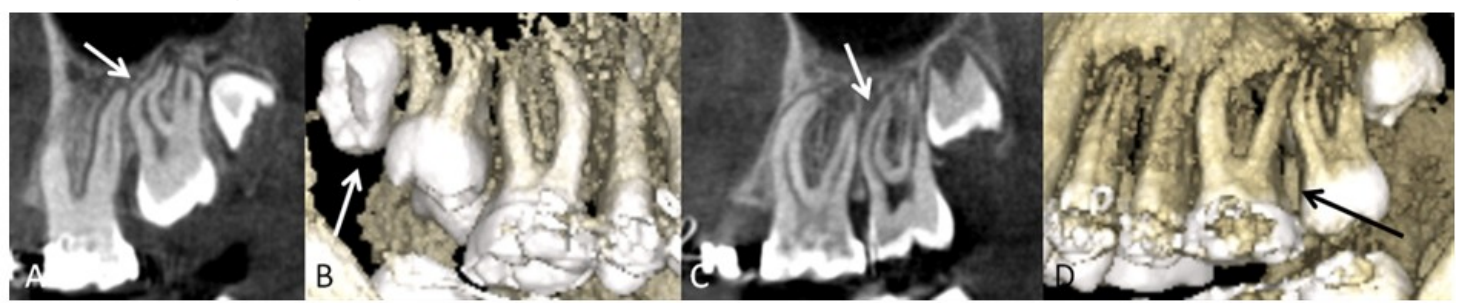

Fig. 9. A. Vue sagittale droite. La deuxième molaire maxillaire droite (dent 17 ) est incluse et présente une dilacération de la racine mésiale (flèche). Le sac folliculaire de cette dent est en contact avec la première molaire maxillaire droite (dent 16). La troisième molaire maxillaire droite (dent 18) est profondément incluse.

B. Reconstruction 3D, vue latérale droite. L'éruption de la dent 17 sur l'arcade prend une direction mésiale (flèche). La dent 18 est vestibuloversée avec sa face occlusale en vestibulaire.

C. Vue sagittale gauche (coté sain). La deuxième molaire maxillaire gauche (dent 27) se trouve sur l'arcade et ne présente pas de dilacération radiculaire. La troisième molaire maxillaire gauche (dent 28 ) est profondément incluse.

D. Reconstruction 3D, vue latérale gauche (côté sain). L'éruption de la dent 27 sur l'arcade prend une direction distale (flèche). 


\section{Résorptions radiculaires externes}

Dans le contexte des traitements orthodontiques, les appareils et les techniques utilisés peuvent parfois induire l'application de forces excessives sur les dents ou des vitesses excessives de déplacement dentaire. Les séquelles de ces évènements indésirables sont l'apparition de résorptions radiculaires externes [13, 14]. Des calcifications intra-pulpaires peuvent également être provoquées par l'application de forces orthodontiques excessives [15]. La figure 10 illustre la résorption radiculaire externe post-traitement orthodontique ainsi que la présence de calcifications intrapulpaires chez une patiente de 34 ans. Sur le plan médico-légal, il serait plus prudent de réaliser un $\mathrm{CBCT}$, chez l'adulte, préalablement à tout traitement orthodontique ou lors d'un changement de praticien.

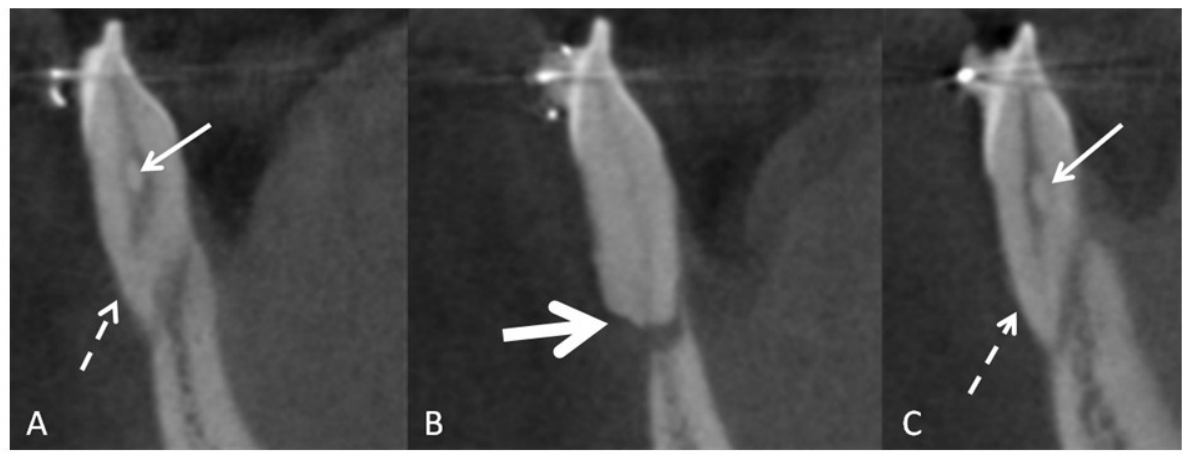

Fig. 10. A. Vue sagittale. La flèche avec tirets indique la résorption radiculaire externe au niveau apical de l'incisive latérale mandibulaire droite (dent 42) et l'absence de la corticale vestibulaire. Une calcification pulpaire est observée au sein de cette même dent (flèche pleine).

B. Vue sagittale. L'incisive centrale mandibulaire droite présente une résorption radiculaire externe horizontale au niveau de l'apex (flèche). II existe également une lyse de la corticale osseuse vestibulaire en regard de cette lésion et une fistule est présente.

C. Vue sagittale. L'absence de la corticale vestibulaire est indiquée par la flèche avec tirets. La flèche pleine montre une calcification intra-pulpaire au sein de l'incisive latérale mandibulaire gauche (dent 32). 


\section{Conclusion}

Comme tout examen d'imagerie médicale, le CBCT doit être justifié individuellement, au cas par cas, et pouvoir apporter un bénéfice au patient en terme de diagnostic et/ou de traitement orthodontique. Le recours systématique à l'examen par CBCT pour tous les patients en vue de ce type de traitement est actuellement considéré comme inadéquat [2].

Il est également important de garder à l'esprit que l'orthodontiste prescripteur doit être capable d'interpréter et est responsable de l'interprétation de tout ce qui est visible sur les clichés radiographiques, soit de l'ensemble du champs de vue [16]. Sur un CBCT, la fréquence des découvertes fortuites indépendantes de l'objet de la demande d'examen est de $25 \%$ [17]. Il est donc primordial que l'orthodontiste soit capable de reconnaître, sur les images du $\mathrm{CBCT}$, les éléments essentiels à son diagnostic et/ou à son traitement mais également tous les autres détails (variantes anatomiques prédisposant à certaines pathologies, découvertes fortuites de pathologies,...) présents dans le champs de vue de l'examen et indépendant de la demande initiale. Une étude américaine réalisée en 2012 par Ahmed et al. a révélé l'absence de diagnostic de $67 \%$ de lésions et le diagnostic de $50 \%$ de faux-positifs lors de l'analyse d'images de CBCT par des orthodontistes et des assistants en formation en orthodontie [18].

L'interprétation correcte des images issues d'un CBCT requiert une formation complète, de qualité et de niveau universitaire, donnée par des experts en CBCT dento-maxillo-facial, accrédités au niveau européen, auprès de leurs agences de contrôles nucléaires respectifs ou autres organes de contrôle et de radioprotection [19]. 
- Remerciements: aucun.

- Sources de financement: cette étude n'a reçu aucun soutien financier.

- Conflit d'intérêt: Pr R. Olszewski est Editeur-en-Chef de Nemesis; les autres auteurs déclarent qu'ils/elles n'ont pas de conflit d'intérêt lié à cette revue illustrée.

- Approbation éthique: cette revue de la littérature illustrée n'a pas nécessité d'approbation de comité éthique.

- Consentement éclairé: ce travail de revue de la littérature illustrée n'a pas nécessité de consentement éclairé de la part de patients. Toutes les images sont anonimisées.

\section{Contribution des auteurs}

\begin{tabular}{|l|l|}
\hline Auteur & Contribution \\
\hline Olszewski Raphael & $\begin{array}{l}\text { Concept, Recherches, Méthodologie, } \\
\text { Données, Supervision, Validation, } \\
\text { Visualisation, Préparation de } \\
\text { manuscrit, Ecriture, Corrections et édition }\end{array}$ \\
\hline Theys Stéphanie & $\begin{array}{l}\text { Validation, Visualisation, Préparation de } \\
\text { manuscrit, Ecriture, Corrections et édition }\end{array}$ \\
\hline Perez Eytan & $\begin{array}{l}\text { Validation, Visualisation, Préparation de } \\
\text { manuscrit, Ecriture, Corrections et édition }\end{array}$ \\
\hline Wisniewska Katarzyna & $\begin{array}{l}\text { Validation, Visualisation, Préparation de } \\
\text { manuscrit, Ecriture, Corrections et édition }\end{array}$ \\
\hline Wisniewski Marcin & $\begin{array}{l}\text { Concept, Validation, Visualisation, } \\
\text { Préparation de manuscrit, Ecriture, } \\
\text { Corrections et édition }\end{array}$ \\
\hline
\end{tabular}




\section{References}

408
1. Scarfe WC, Azevedo B, Toghyani S, Farman AG. Cone Beam Computed Tomographic imaging in orthodontics. Aust Dent J 2017;62:33-50.

2. Abdelkarim A. Cone-Beam Computed Tomography in orthodontics. Dent $\mathbf{J}$ (Basel) 2019;7:89.

3. Oenning AC, Jacobs R, Pauwels R, Stratis A, Hedesiu M, Salmon B, DIMITRA Research Group, http://www.dimitra.be. Cone-beam CT in paediatric dentistry: DIMITRA project position statement. Pediatr Radiol 2018;48:308-316.

4. Colceriu-Şimon IM, Băciuț M, Stiufiuc RI, Aghiorghiesei A, Țărmure V, Lenghel M, Hedeşiu M, Băciuţ G. Clinical indications and radiation doses of cone beam computed tomography in orthodontics. Med Pharm Rep 2019;92:346-351.

5. De Grauwe A, Ayaz I, Shujaat S, Dimitrov S, Gbadegbegnon L, Vande Vannet B, Jacobs R. CBCT in orthodontics: a systematic review on justification of CBCT in a paediatric population prior to orthodontic treatment. Eur J Orthod 2019;41:381389.

6. Palomo JM, Rao PS, Hans MG. Influence of CBCT exposure conditions on radiation dose. Oral Surg Oral Med Oral Pathol Oral Radiol Endod 2008;105:773782.

7. Wriedt S, Jaklin J, Al-Nawas B, Wehrbein H. Impacted upper canines: examination and treatment proposal based on $3 \mathrm{D}$ versus $2 \mathrm{D}$ diagnosis. J Orofac Orthop 2012; 73:28-40.

8. Alqerban A, Jacobs R, Fieuws S, Willems G. Radiographic predictors for maxillary canine impaction. Am J Orthod Dentofacial Orthop 2015;147:345-354.

9. Liu DG, Zhang WL, Zhang ZY, Wu YT, Ma XC. Localization of impacted maxillary canines and observation of adjacent incisor resorption with cone-beam computed tomography. Oral Surg Oral Med Oral Pathol Oral Radiol Endod 2008;105:91-98.

10. Liu DG, Zhang WL, Zhang ZY, Wu YT, Ma XC. Three-dimensional evaluations of supernumerary teeth using cone-beam computed tomography for 487 cases. Oral Surg Oral Med Oral Pathol Oral Radiol Endod 2007;103:403-411. 
11. Gurler G, Delilbasi C, Delilbasi E. Investigation of impacted supernumerary teeth: a cone beam computed tomograph (cbct) study. J Istanb Univ Fac Dent 2017;51:18-24.

12. Goksel S, Agirgol E, Karabas HC, Ozcan I. Evaluation of prevalence and positions of mesiodens using Cone-Beam Computed Tomography. J Oral Maxillofac Res 2018;9:e1.

13. Castro IO, Alencar AH, Valladares-Neto J, Estrela C. Apical root resorption due to orthodontic treatment detected by cone beam computed tomography. Angle Orthod 2013;83:196-203.

14. Deng Y, Sun Y, Xu T. Evaluation of root resorption after comprehensive orthodontic treatment using cone beam computed tomography (CBCT): a metaanalysis. BMC Oral Health 2018;18:116.

15. Han G, Hu M, Zhang Y, Jiang H. Pulp vitality and histologic changes in human dental pulp after the application of moderate and severe intrusive orthodontic forces. Am J Orthod Dentofacial Orthop 2013;144:518-522.

16. Friedland B. Medicolegal issues related to cone beam CT. Semin Orthod 2009;15:77-84.

17. Cha JY, Mah J, Sinclair P. Incidental findings in the maxillofacial area with 3 dimensional cone-beam imaging. Am J Orthod Dentofacial Orthop 2007;132:7-14.

18. Ahmed F, Brooks SL, Kapila SD. Efficacy of identifying maxillofacial lesions in cone-beam computed tomographs by orthodontists and orthodontic residents with third-party software. Am J Orthod Dentofacial Orthop 2012; 141:451-459.

19. Brown J, Jacobs R, Levring Jäghagen E, Lindh C, Baksi G, Schulze D, Schulze $\mathrm{R}$, European Academy of DentoMaxilloFacial Radiology. Basic training requirements for the use of dental CBCT by dentists: a position paper prepared by the European Academy of DentoMaxilloFacial Radiology. Dentomaxillofac Radiol 2014;43:20130291. 